Communications in Physics, Vol.21, No. 1 (2011), pp. 11-18

\title{
SPARTICLE MASSES IN A SUPERSYMMETRIC GRAND UNIFIED MODEL
}

\author{
TRAN MINH HIEU \\ Institute of Engineering Physics, Hanoi University of Science and Technology \\ and \\ Faculty of Physics, Hanoi University of Science, Vietnam National University, Hanoi
}

\begin{abstract}
In this paper, we investigate the phenomenological aspect relevant to the sparticle masses of a supersymmetric grand unified model, in which the $S U(5)$ is used as the grand unified group with the minimal particle contents and supersymmetry breaking is mediated by the so-called gaugino mediation mechanism. A detailed analysis about the dependence of the squarks and sleptons masses on the free parameter space is given, and shown to be useful for the collider phenomenological study and parameter determination for future experiments.
\end{abstract}

\section{INTRODUCTION}

Supersymmetry (SUSY) is well-known as a promising solution to a long-standing problem in the Standard Model (SM), the fine-tuning problem. Recently, with the possibility of being tested at the Large Hardon Collider (LHC) and other future collider projects such as the International Linear Collider (ILC), lots of attentions have been focused on this symmetry. Moreover, in the present of R-parity conservation, the lightest supersymmetric particle (LSP) in the minimal supersymmetric extension of the SM (MSSM) is stable, electrically neutral and weakly interacting particle which is a good candidate for the dark matter necessary to explain the cosmological observation. Another attractive point of this model is that in the MSSM all the couplings naturally unify at the scale $M_{G U T}$ of the order $10^{16} \mathrm{GeV}$ giving a hint for a grand unified theory (GUT).

SUSY predicts that the SM particles and their superpartners must have equal masses. However, we have not seen any signal of such sparticles in both direct and indirect searches so far. It implies that SUSY must be broken in a certain way which suppresses the flavour changing neutral currents (FCNC). To do so, many different mechanisms have been proposed. But they all have a general feature that the SUSY breaking sector needs to be mediated to the MSSM sector via a flavour blind interaction to produce soft terms in the Lagrangian.

In this paper, we concentrate on a possibility so-called gaugino mediation $[1,2]$. With the extra-dimensional configuration, the SUSY breaking source in this scenario is mediated to the matter sector by transferring gauge supermultiplet in the bulk. This setup ensures finite masses for gauginos, negligible sfermion masses and trilinear couplings at the compactification scale. At low energy scale, the sfermion masses and trilinear couplings acquire their values through renormalization group (RG) runnings with the flavour blind 
gauge interactions. This fact enables our theories to suppress the FCNCs. Nevertheless, the cosmological constraint on the dark matter turns out to conflict with the mass spectrum predicted by this model. This fact, known as $\tilde{\tau}$-LSP problem, leads to the necessity of pushing up the compactification scale to be higher than the GUT scale. It follows that in gaugino mediation we need to consider SUSY GUT models instead of just the MSSM. Here, we take the $S U(5)$ grand unified gauge group with minimal particle contents for a concrete study. Because of the higher-dimensional origin and the gauge unification, the number of free parameters in the gaugino mediation reduces significantly making our models very predictive $[3,4]$. This advantage of the model enables us to examine in detail the sparticle masses in relation with the free parameters.

The paper is organized as follows: In Section 2, we briefly review the gaugino mediation and discuss the $\tilde{\tau}$-LSP problem in this scenario. The parameter dependences of sparticle masses in the minimal $S U(5)$ GUT model with gaugino-mediated SUSY breaking are presented in Section 3. Finally, we conclude in the last Section.

\section{GAUGINO MEDIATION AND $\tilde{\tau}$-LSP PROBLEM}

Gaugino mediation scenario bases on a 5-dimensional space-time setup in which the extra-dimension is compactified on a $S^{1} / Z_{2}$ orbifold. The SUSY breaking sector stays in a $(3+1)$-dimensional brane and the MSSM matter sector in another one. These two branes locate at two different fixed points in the fifth dimension, i.e. $x_{5}=0, \pi$. When there is no interaction in the fifth dimension, such geometrical separation makes sure that the matter sector is exactly supersymmetric. The soft terms can be obtained by supposing the gauge supermultiplet propagates in the bulk between the two branes. The SUSY breaking source couples directly to gauginos producing their masses at tree level. The distance in the extra-dimension protects the two sectors from interacting at tree level giving zero soft masses at the compactification scale $M_{c}$. The sparticles acquire soft masses at ordinary scale only through the RG evolution. Similarly, the third type of soft SUSY breaking terms, the scalar trilinear couplings, is also suppressed at $M_{c}$.

Assuming the gauge couplings unify at some scale larger than the compactification scale, the gaugino masses satisfy the relation as follows:

$$
\frac{M_{1}}{g_{1}^{2}}=\frac{M_{2}}{g_{2}^{2}}=\frac{M_{3}}{g_{3}^{2}}=\frac{M_{u}}{g_{u}^{2}}
$$

at any scale. The above relation helps to reduce the number of free parameters in gaugino mediation to only three plus a sign:

$$
M_{1}^{c}, M_{c}, \tan \beta, \operatorname{sign}(\mu),
$$

where $M_{1}^{c}, \tan \beta, \mu$ is the $U(1)_{Y}$ gaugino mass at $M_{c}$, the ratio of the vacuum expectation values of two Higgs doublets, and the supersymmetric Higgs mass respectively. Within the MSSM framework, we input the gaugino mass at the compactification scale, then solve the renormalization group equations (RGEs) [5] from $M_{c}$ toward low energies. To obtain the mass eigenstates of stau and neutralino, we diagonalize the mass matrices for stau in 
left-right basis [6]:

$$
M_{\tilde{\tau}}^{2}=\left(\begin{array}{cc}
m_{\tilde{L}_{3}}^{2}+m_{\tau}^{2}-\left(\frac{1}{2}-\mathrm{s}_{\mathrm{w}}{ }^{2}\right) M_{Z}^{2} \cos 2 \beta & m_{\tau}\left(A_{\tau}-\mu \tan \beta\right) \\
m_{\tau}\left(A_{\tau}-\mu \tan \beta\right) & m_{\tilde{E}_{3}}^{2}+m_{\tau}^{2}-\mathrm{s}_{\mathrm{w}}{ }^{2} \cos 2 \beta
\end{array}\right),
$$

and for neutralino [7]:

$$
M_{\tilde{\chi}_{1}^{0}}^{2}=\left(\begin{array}{cccc}
M_{1} & 0 & -M_{Z} \mathrm{~s}_{\mathrm{w}} \cos \beta & M_{Z} \mathrm{~s}_{\mathrm{w}} \sin \beta \\
0 & M_{2} & M_{Z} \mathrm{c}_{\mathrm{w}} \cos \beta & -M_{Z} \mathrm{c}_{\mathrm{w}} \sin \beta \\
-M_{Z} \mathrm{~s}_{\mathrm{w}} \cos \beta & M_{Z} \mathrm{c}_{\mathrm{w}} \cos \beta & 0 & -\mu \\
M_{Z} \mathrm{~s}_{\mathrm{w}} \sin \beta & -M_{Z} \mathrm{c}_{\mathrm{w}} \sin \beta & -\mu & 0
\end{array}\right),
$$

in which $\mathrm{s}_{\mathrm{w}}=\sin \theta_{W}$ and $\mathrm{c}_{\mathrm{w}}=\cos \theta_{W}$, with $\theta_{W}$ being the weak mixing angle.

In Fig. 1, we plot the lighter stau and the lightest neutralino masses as functions of the compactification scale with $M_{1}^{c}=400 \mathrm{GeV}, \tan \beta=10$ and $\operatorname{sign} \mu=+1$. From Fig. 1a, we see that the neutralino mass (the dashed line) is always larger than the stau mass (the solid line) when $M_{c}<M_{G U T}$. Therefore the stau is the LSP which is stable and would be the final product of all processes involving sparticles in theories with Rparity conservation. From the cosmological point of view, a SUSY model with $\tilde{\tau}$-LSP is problematic because such charged particle cannot be a candidate for dark matter.

(a)

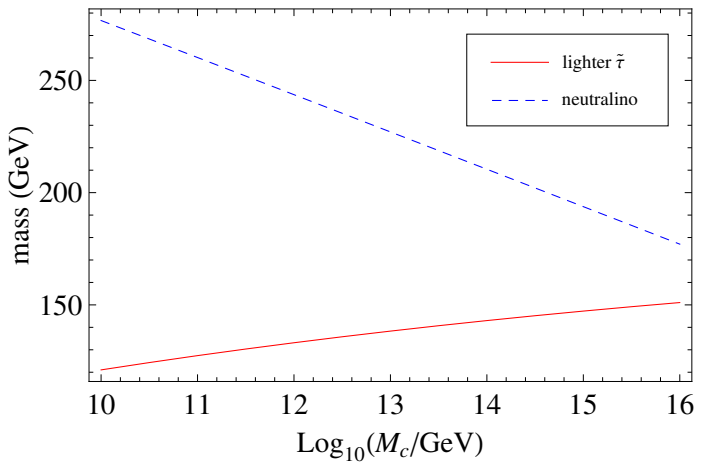

(b)

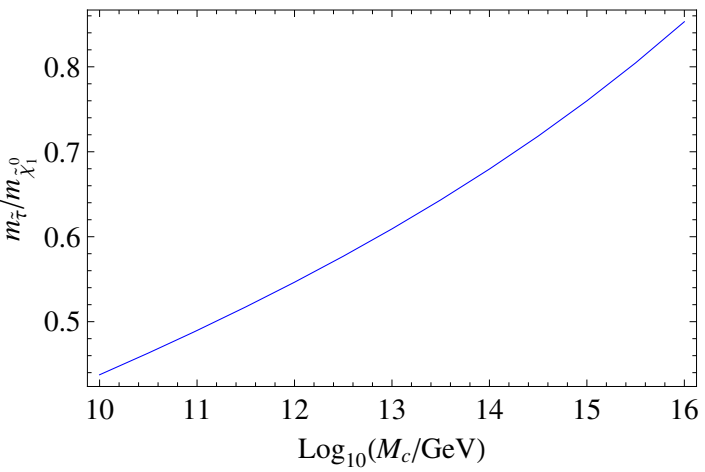

Fig. 1. The dependences on the compactification scale of: (a) the lighter stau and the lightest neutralino masses, (b) the mass ratio between the lighter stau and the lightest neutralino in the MSSM when $M_{c}<M_{G U T}, M_{1}^{c}=400 \mathrm{GeV}, \tan \beta=10$ and $\operatorname{sign} \mu=+1$.

However, when increasing $M_{c}$, the mass gap between these two particles is narrower, namely the mass ratio between them raises up as we can see in Fig. 1b. The ratio approximately reaches 0.84 which is close to 1 if we choose $M_{c}=10^{16} \mathrm{GeV}$. This fact gives us a hint to solve the $\tilde{\tau}$-LSP problem by pushing up the compactification scale to be higher than the GUT scale. In this case, a unified gauge group with a specific particle content need to be clarified since we deal with a SUSY GUT model. 


\section{SPARTICLE MASSES IN THE MINIMAL $S U(5)$ GUT WITH GAUGINO MEDIATED SUSY BREAKING}

\section{Solution to the $\tilde{\tau}$-LSP Problem}

In the minimal $S U(5)$ model, the particle content is organized as follows $[8,9,10]$ : $D_{i}^{c}$ and $L_{i}$ realize the $\overline{\mathbf{5}}_{i}$ representation, while $Q_{i}, U_{i}^{c}$ and $E_{i}^{c}$ realize the $\mathbf{1 0}_{i}$ representation. The $\overline{\mathbf{5}}_{H}$ and $\mathbf{5}_{H}$ contain the two Higgs doublets needed to break the electroweak symmetry. The other Higgs fields necessary for the grand unification breaking realize the $\mathbf{2 4}_{H}$ representation of the $S U(5)$ group. Since above the GUT scale the normal gauge groups of the MSSM $S U(3)_{C} \times S U(2)_{L} \times U(1)_{Y}$ unify into a single group $S U(5)$, the gauginos also have a common mass in this extremely high energy region. With the gaugino mediated SUSY breaking, the number of free parameters is also three plus a sign:

$$
m_{1 / 2}, M_{c}, \tan \beta, \operatorname{sign}(\mu),
$$

where $m_{1 / 2}$ is the common gaugino mass at the GUT scale.

To generate the mass spectrum, after solving the RG equations of the $S U(5)$ SUSY GUT model from the compactification scale to the GUT scale $[3,11]$, the values of soft terms are determined at $M_{G U T}$ as follows:

$$
\begin{aligned}
& m_{\mathbf{1 0}}^{2}\left(M_{G U T}\right)=\frac{12}{5} m_{1 / 2}^{2}\left[1-\left(\frac{\alpha\left(M_{c}\right)}{\alpha\left(M_{G U T}\right)}\right)^{2}\right] \\
& m_{\overline{\mathbf{5}}}^{2}\left(M_{G U T}\right)=m_{\mathbf{5}}^{2}\left(M_{G U T}\right)=\frac{8}{5} m_{1 / 2}^{2}\left[1-\left(\frac{\alpha\left(M_{c}\right)}{\alpha\left(M_{G U T}\right)}\right)^{2}\right] \\
& A_{u}\left(M_{G U T}\right)=-\frac{32}{5} m_{1 / 2}\left[1-\left(\frac{\alpha\left(M_{c}\right)}{\alpha\left(M_{G U T}\right)}\right)\right] \\
& A_{d}\left(M_{G U T}\right)=-\frac{28}{5} m_{1 / 2}\left[1-\left(\frac{\alpha\left(M_{c}\right)}{\alpha\left(M_{G U T}\right)}\right)\right]
\end{aligned}
$$

where $\alpha$ is the GUT gauge coupling and

$$
\alpha\left(M_{c}\right)^{-1}=\alpha\left(M_{G U T}\right)^{-1}-\frac{3}{2 \pi} \ln \left(M_{G U T} / M_{c}\right) .
$$

Subsequently, the MSSM RG equations are solved from the GUT scale to the electroweak scale with the soft term inputs at $M_{G}$.

In Fig. 2, we plot the masses of stau and neutralino as functions of the compactification scale $M_{c}$. Fig. 2a shows that when $M_{c} \gtrsim 10^{16.5} \mathrm{GeV}$ the stau is heavier than the neutralino and hence we have $\tilde{\chi}_{1}^{0}$-LSP. So by inserting the running above the GUT scale with a grand unified gauge group, the $\tilde{\tau}$-LSP problem has been solved completely. Similar to Fig. 1b, in Fig. 2b, the dependence on the compactification scale of the mass ratio between these particles is presented. We see that when increasing $M_{c}$, not only neutralino becomes LSP, but also the mass discrepancy between the LSP and the next-to-LSP (namely, the stau) also goes up.

However, it is worth to mention that $\tan \beta$ cannot be too large when fixing $m_{1 / 2}$ and $M_{c}$, otherwise our theory would reintroduce the $\tilde{\tau}$-LSP again. For example, choosing 
(a)

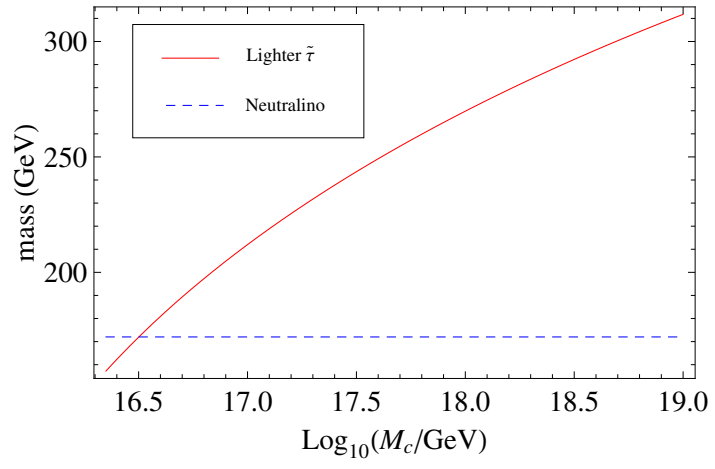

(b)

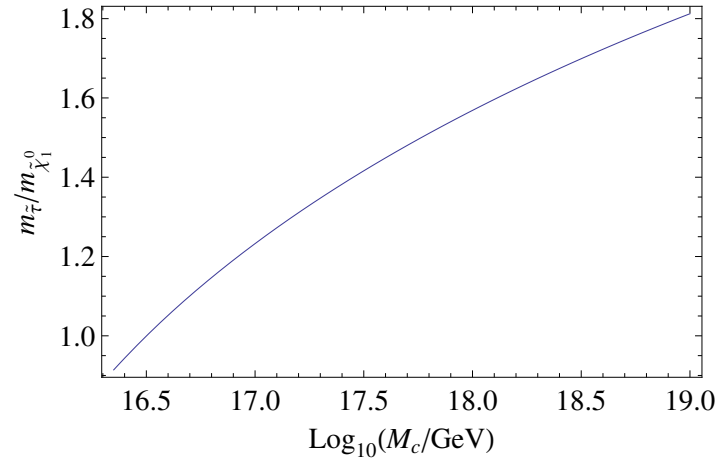

Fig. 2. The dependences on the compactification scale of: (a) the lighter stau and the lightest neutralino masses, (b) the mass ratio between the lighter stau and the lightest neutralino in the minimal $S U(5)$ SUSY GUT when $M_{c}>M_{G U T}$, $m_{1 / 2}=400 \mathrm{GeV}, \tan \beta=10$ and $\operatorname{sign} \mu=+1$.

$m_{1 / 2}=400 \mathrm{GeV}, M_{c}=10^{18} \mathrm{GeV}$ and $\operatorname{sign} \mu=+1$ as in Fig. 3, tan $\beta$ must be smaller than about 44 . That means, for given values of $m_{1 / 2}$ and $M_{c}$, there exits an upper limit for $\tan \beta$.

(a)

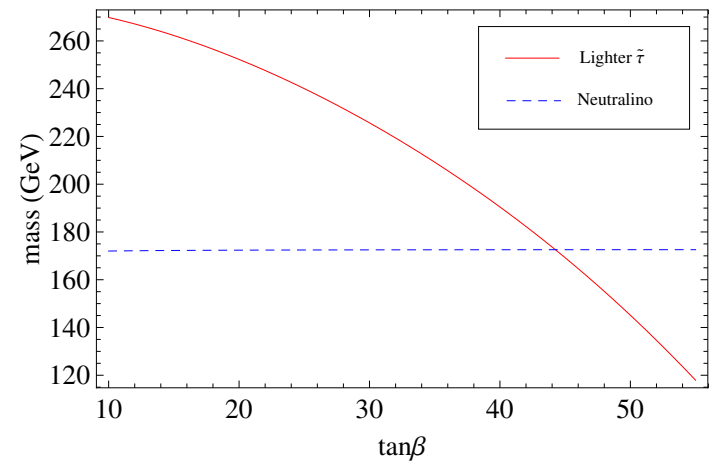

(b)

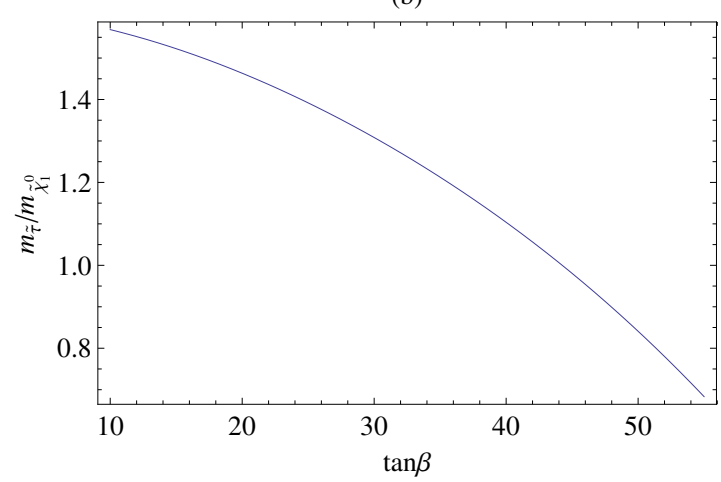

Fig. 3. The dependences on $\tan \beta$ of: (a) the lighter stau and the lightest neutralino masses, (b) the mass ratio between the lighter stau and the lightest neutralino in the minimal $S U(5)$ SUSY GUT when $M_{c}>M_{G U T}, m_{1 / 2}=400 \mathrm{GeV}$, $M_{c}=10^{18}$ and $\operatorname{sign} \mu=+1$.

\section{Sparticle Masses Analysis}

Doing the analogous things to the LSP analysis, now we vary only one of the three parameters and keep the others fixed to see the effects of the input parameters on the sparticle masses. In Fig. 4, we plot the dependences of the first generation sparticles masses on the input paramters, while those of the third ones are shown in Fig. 5. Since 
the second generation almost degenerates with the first one, its parameter dependences are also the same as in Fig. 4.

(a)

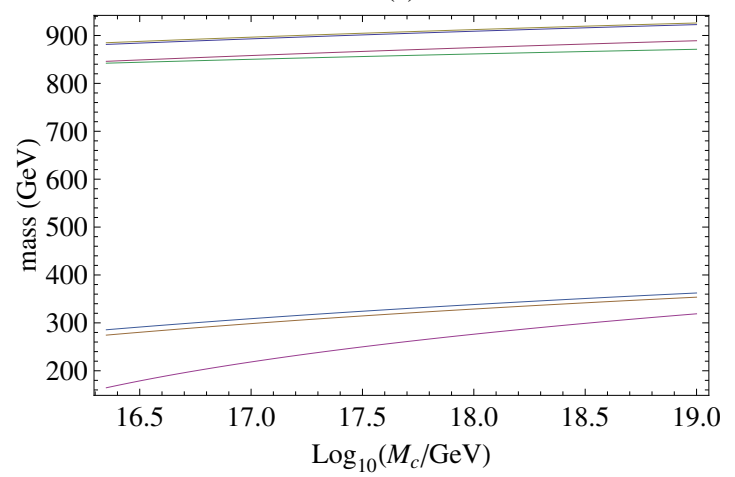

(b)

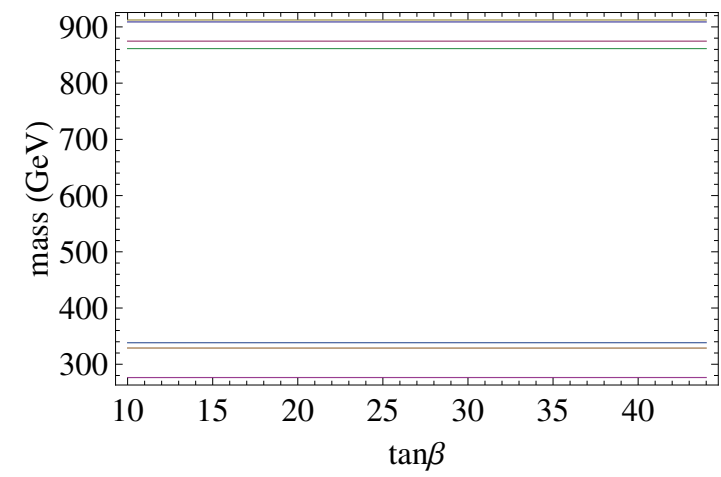

(c)

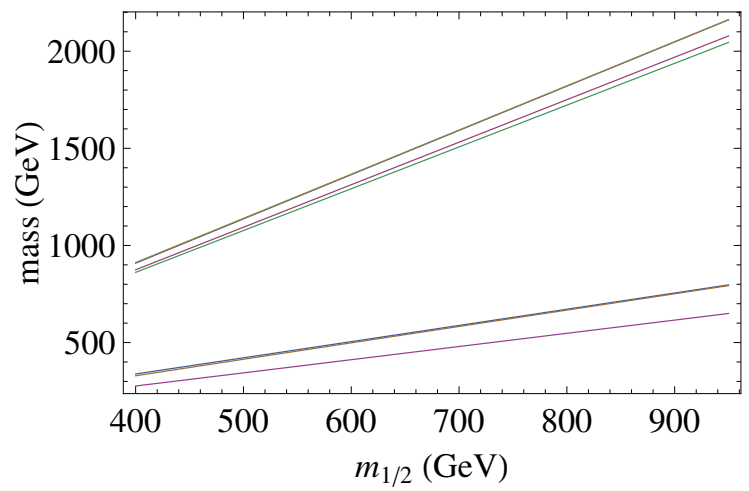

Fig. 4. The masses of sparticles of the first (two) generation(s) as functions of: (a) the compactification scale $M_{c}$ with $m_{1 / 2}=400 \mathrm{GeV}$ and $\tan \beta=10$, (b) $\tan \beta$ with $m_{1 / 2}=400 \mathrm{GeV}$ and $M_{c}=10^{18} \mathrm{GeV}$, (c) $m_{1 / 2}$ with $M_{c}=10^{18}$ and $\tan \beta=10$. In all plots, from bottom to top, the lines correspond to $m_{\tilde{e}_{2}}, m_{\tilde{\nu}_{e}}$, $m_{\tilde{e}_{1}}, m_{\tilde{d}_{2}}, m_{\tilde{u}_{2}}, m_{\tilde{u}_{1}}$ and $m_{\tilde{d}_{1}}$. 
(a)

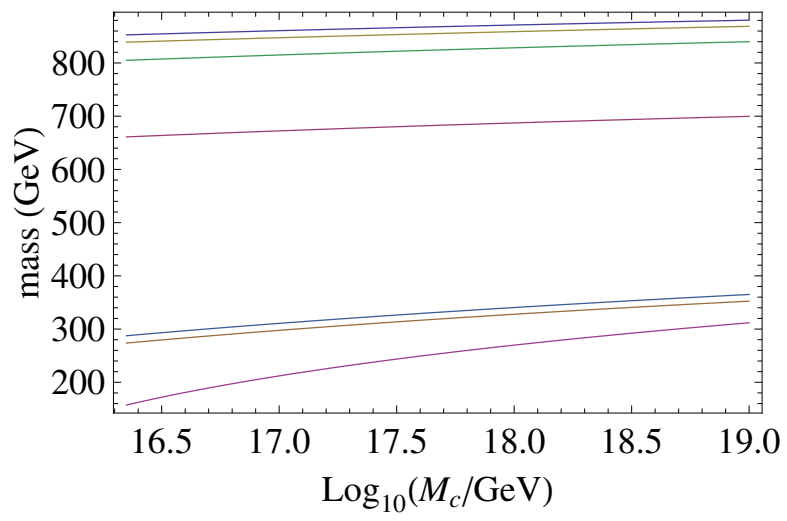

(b)

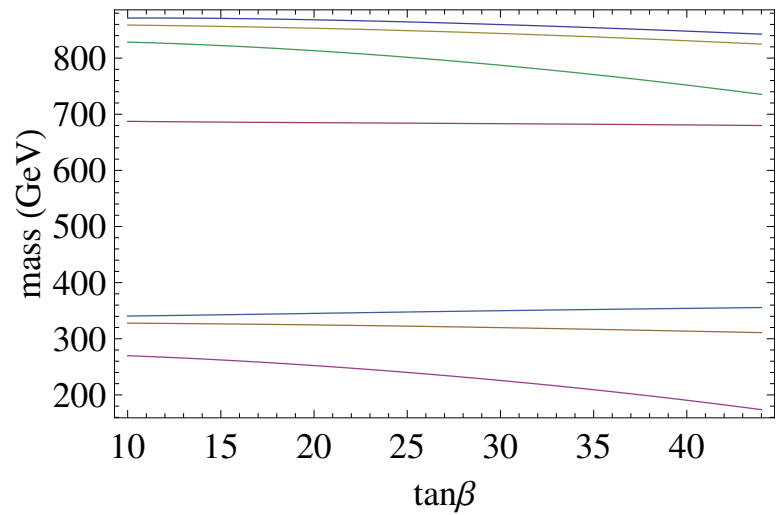

(c)

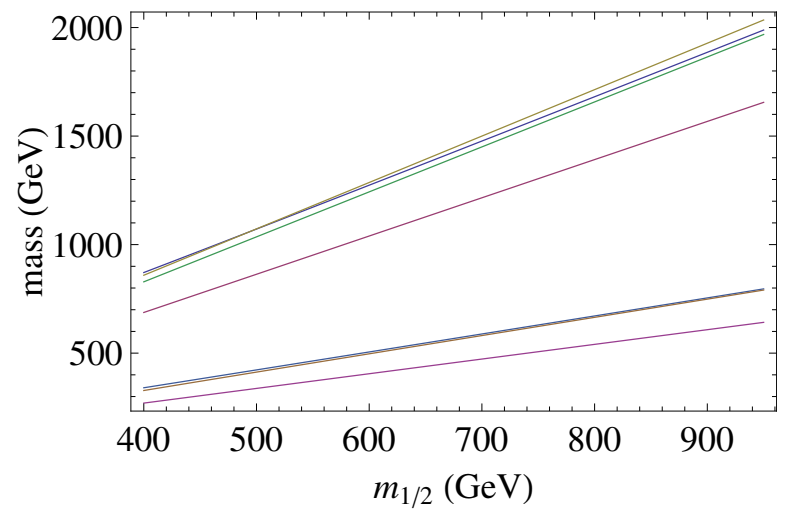

Fig. 5. The masses of sparticles of the third generation as functions of: (a) the compactification scale $M_{c}$ with $m_{1 / 2}=400 \mathrm{GeV}$ and $\tan \beta=10$, (b) $\tan \beta$ with $m_{1 / 2}=400 \mathrm{GeV}$ and $M_{c}=10^{18} \mathrm{GeV}$, (c) $m_{1 / 2}$ with $M_{c}=10^{18}$ and $\tan \beta=10$. In all plots, at $m_{1 / 2}=400 \mathrm{GeV}$, from bottom to top, the lines correspond to $m_{\tilde{\tau}_{2}}$, $m_{\tilde{\nu}_{\tau}}, m_{\tilde{\tau}_{1}}, m_{\tilde{t}_{2}}, m_{\tilde{b}_{2}}, m_{\tilde{b}_{1}}$ and $m_{\tilde{t}_{1}}$. 
TRAN MINH HIEU

In the both figures, we see that the mass hierarchy between squarks and sleptons is respected, namely the squarks are always heavier than the sleptons. Concentrating on the squark sector of the model, the mass splitting in the third generation is larger than that in the first two generations. It is due to the contribution of the top Yukawa coupling. Except the case of Fig. $4 \mathrm{~b}$ where the sfermion masses of the first two generations are almost unchanged with respect to $\tan \beta$, the sparticle masses are slightly changed with respect to the compactification scale $M_{c}$ and $\tan \beta$ as presented in Figs. 4a, 5a and 5b. Nevertheless, the effect of the remaining free parameter, namely the gaugino mass at the GUT scale $m_{1 / 2}$, is more crucial. In Figs. $4 \mathrm{c}$ and $5 \mathrm{c}$, we see that the larger $m_{1 / 2}$ can significantly drive sparticle masses up.

\section{CONCLUSION}

In the framework of gaugino mediation scenario, we have considered the minimal $S U(5)$ SUSY GUT as the solution to the $\tilde{\tau}$-LSP problem of the ordinary MSSM. We have pointed out that, for given values of the compactification scale and $m_{1 / 2}$, this solution only applies to a certain range of $\tan \beta$ whose upper limit is set. By varying the input parameters one by one, we have shown the their effects on the sparticle masses. The results here can be used in collider phenomenological study and paramter determination in experiments, such as the Large Hardron Collider and the future International Linear Collider.

\section{ACKNOWLEDGMENT}

The author would like to thank Prof. Nobuchika Okada for the fruitful discussions. This work is supported in part by the project T2010-195, HUST.

\section{REFERENCES}

[1] D. E. Kaplan, G. D. Kribs and M. Schmaltz, Phys. Rev. D62 (2000) 035010.

[2] Z. Chacko, M. A. Luty, A. E. Nelson and E. Ponton, JHEP 0001 (2000) 003.

[3] M. Schmaltz and W. Skiba, Phys. Rev. D62 (2000) 095004.

[4] M. Schmaltz and W. Skiba, Phys. Rev. D62 (2000) 095005.

[5] D. J. Castano, E. J. Piard and P. Ramond, Phys. Rev. D49 (1994) 4882.

[6] W. Hollik and H. Rzehak, Eur. Phys. J. C32 (2003) 127.

[7] M. M. E. Kheishen, A. A. Shafik and A. A. Aboshousha Phys. Rev. D45 (1992) 4345

[8] H. Georgi and S. L. Glashow Phys. Rev. Lett. 32 (1974) 438.

[9] S. Dimopoulos and H. Georgi, Nucl. Phys. B193 (1981) 150.

[10] N. Sakai, Z. Phys. C11 (1981) 153.

[11] N. Okada and H. M. Tran, Phys. Rev. $\mathbf{D 8 3}$ (2011) 053001. 